\title{
Leid und Hoffnung einer Nation im Graffiti
}

\author{
Hannah Griese
}

In Zeiten der Corona-Krise gewinnt die Idee der Nation an Bedeutung: Grenzen werden geschlossen, jeder Staat hilft zunächst vor allem sich selbst. Politiker:innen und Medien appelieren an die Solidarität der Bürger:innen und inszenieren die Nation als eine Leidens- und Schicksalsgemeinschaft, die in der Krise zusammenhält. Medien spielen eine wichtige Rolle bei der Konstruktion von nationalen Gemeinschaften, indem sie Gefühle der Gemeinsamkeit und Zusammengehörigkeit vermitteln. Auch während der Pandemie thematisieren verschiedene mediale Gattungen (nationalen) Zusammenhalt, wobei sie mitunter auf religiöse Motive zurückgreifen. Dieser Beitrag widmet sich einer besonderen Art von Bildern, dem Graffiti.

Rund um die Welt entstehen zurzeit zahlreiche Streetart-Werke, die sich mit der Corona-Krise auseinandersetzen. Immer wieder finden sich dabei Darstellungen von (vorwiegend weiblichem) Krankenhauspersonal, wobei häufig auf religiöse Symbole und Motive zurückgegriffen wird. Diese Kunstwerke, die im weitesten Sinne des Begriffs als «Graffitis» bezeichnet werden können, befinden sich im öffentlichen Raum, doch ihre große Reichweite erhalten sie durch ihre mediale Verbreitung: Sie werden fotografiert, über soziale Medien, Zeitungen oder Nachrichtenkanäle verbreitet und erreichen somit ein globales Publikum.

Ende April 2020 wurde die Fotografie eines Graffitis aus der norditalienischen Stadt Bergamo auf Twitter gepostet (Abb. 10). Es zeigt eine Frau in Krankenhauskleidung und mit Flügeln, die den kartografischen Umriss Italiens in den Armen hält. Dabei handelt es sich um die Vergrößerung eines digitalen Ölgemäldes des italienischen Künstlers Franco Rivolli mit dem Namen Angels, das auch unter dem Titel La dottoressa che culla l'Italia (Die Ärztin, die Italien wiegt) bekannt geworden ist. Das Poster befindet sich an der Fassade des Papa Giovanni XXIII. Krankenhauses in Bergamo. Es erreichte insbesondere in Italien große Beachtung. 


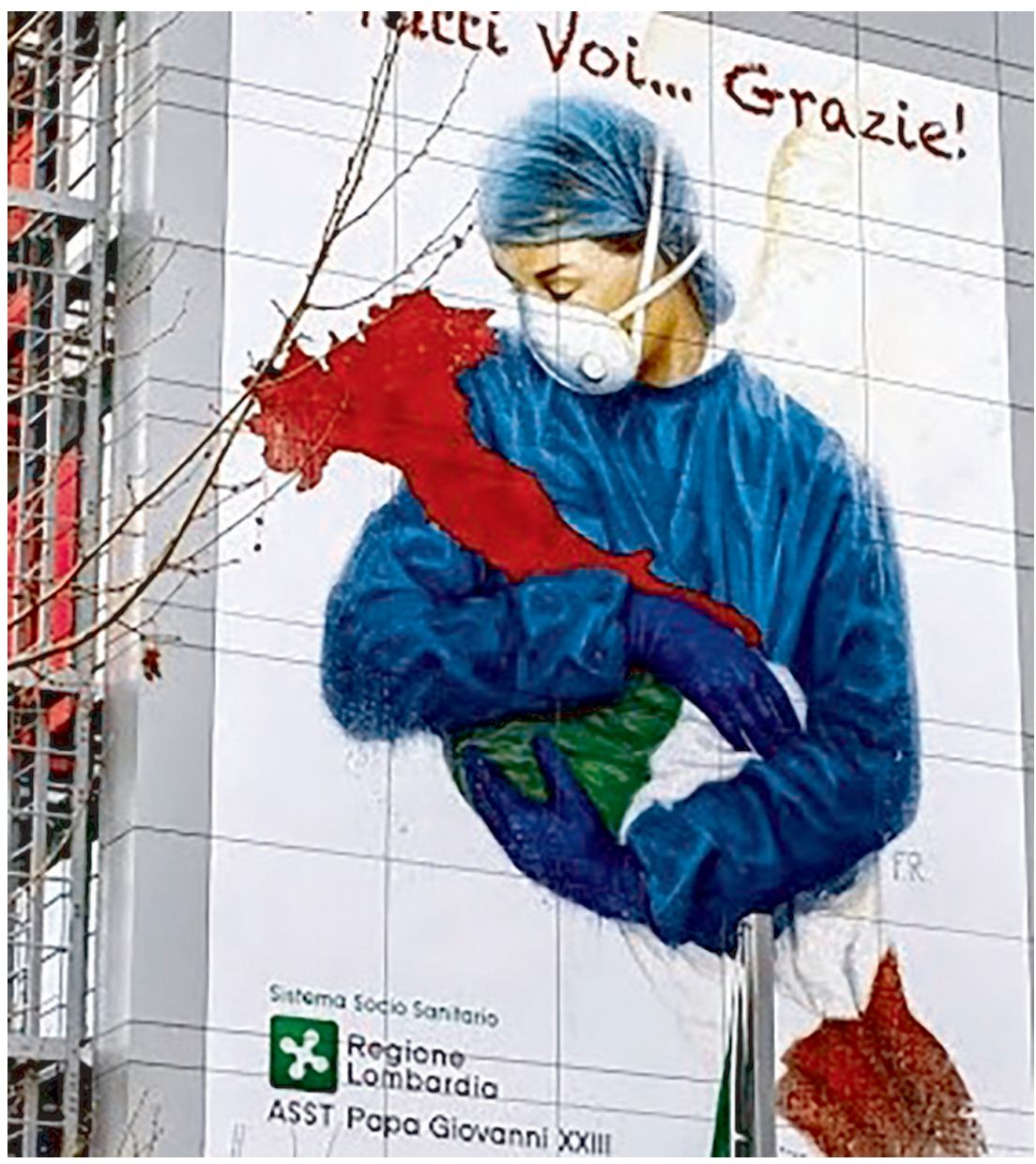

Abb. 10: La dottoressa che culla l'Italia, Vergrößerung eines Werkes von Franco Rivolli an der Wand des Papa Giovanni XXIII. Krankenhauses in Bergamo.

Die meisten Menschen - wie auch ich - sehen nicht das originale Graffiti unmittelbar vor Ort, sondern eine Reproduktion, die zum Beispiel über soziale Medien verbreitet wurde. Das ist eine wichtige Bemerkung, weil die zirkulierenden Fotografien des Werkes einen spezifischen Blick auf das Kunstwerk, eine bestimmte Perspektive sowie einen ausgewählten Ausschnitt vorgeben. Die Reproduktion ist somit selektiv und beeinflusst unsere Wahrnehmung des Graffitis erheblich. So wird die Wirkung beispiels- 
weise dadurch verändert, dass wir das Wandbild auf dem Foto viel kleiner sehen, als es in Wirklichkeit ist, wodurch es weniger eindrucksvoll wirkt. Auch ist eine Fotografie zweidimensional und fixiert die Distanz der Betrachtung sowie Größen-, Farb- und Lichtverhältnisse. Der Blick auf das Kunstwerk ist den Rezipierenden somit vorgegeben. Wie die Betrachter:innen blicke ich in dieser Analyse durch die fotografische Reproduktion auf die außermediale Wirklichkeit, das eigentliche Graffiti an seinem eigentlichen Ort, dem Krankenhaus in Bergamo.

\section{Zwischen Schutz und Apokalypse}

Das Wandbild zeigt eine junge Frau in blauem Kittel mit Handschuhen, Haube und Mundschutz, die die rot gefärbte Silhouette sowie die Flagge Italiens in den Armen hält. Am Rücken der Frau befinden sich helle Flügel. Über ihrem Kopf sind die Worte «A Tutti Voi... Grazie!» («Euch allen... Danke!») geschrieben, in der unteren linken Ecke befindet sich das Logo des Papa Giovanni XXIII. Krankenhauses. Allem Anschein nach gehört die Frau zum Krankenhauspersonal - der Titel des Bildes weist sie als Ärztin aus, der Darstellung nach könnte es auch eine Krankenpflegerin sein. Sie hält die italienische Nation, symbolisiert durch Silhouette und Flagge, in den Armen, wie eine Mutter ihr kleines Kind.

Das Bild enthält verschiedene Verweise auf die christliche Tradition. Ein solches Vorkommen religiöser Motive außerhalb dezidiert religiöser Kunst und außerhalb der Einflussbereiche religiöser Institutionen ist dabei nicht ungewöhnlich, man denke nur an Bereiche wie Werbung oder Spielfilme. Die Symbole werden dabei zwar aus dem Repertoire der jeweiligen Tradition entnommen, jedoch individuell weiterentwickelt, miteinander sowie mit anderen Bildelementen verknüpft oder gar umgedeutet, indem beispielsweise ein Bezug zu aktuellen gesellschaftspolitischen Kontexten hergestellt wird.

Einen sehr expliziten Verweis im vorliegenden Graffiti stellen zunächst die Flügel dar, die auf Engelsfiguren verweisen. In der christlichen Tradition sind Engel übernatürliche Wesen, die im Dienste Gottes stehen. Sie können unterschiedliche Funktionen übernehmen und als Boten auftreten oder als Schutzengel einzelner Menschen und ganzer Völker fungieren. Sie geleiten aber auch die Seelen der Verstorbenen ins Jenseits, vollstrecken die Urteile Gottes und erscheinen am Tag des Jüngsten Gerichts.

Schon hier wird eine Zweideutigkeit der Darstellung ersichtlich: Einerseits stellt das Wandbild ganz klar die als Engel stilisierte Ärztin als Retterfigur und als Beschützerin der italienischen Nation dar. Andererseits findet 
sich hier ein apokalyptisches Moment, indem die Engelsfigur auch als Anspielung auf den Tag des Jüngsten Gerichts, auf ein Urteil Gottes oder auf das Sterben gelesen werden kann. Diese Zweideutigkeit wird durch den möglichen Verweis auf die «apokalyptische Himmelsfrau», einem wiederkehrenden Motiv christlicher Kunst, verstärkt. Im 12. Kapitel der Johannesapokalypse, des letzten Buches der Bibel, wird beschrieben, wie Gott einer bedrohten, kurz vor der Geburt ihres Sohnes stehenden Frauenfigur Flügel verleiht, damit sie in die Wüste fliehen und sich vor einem Drachen retten kann. In der Rezeption dieses Textes wurde diese Figur mit der Mutter Jesu identifiziert. Vor dem Hintergrund dieser Verdichtungen und Überlappung von Anspielungen und Motiven sind unterschiedliche Deutungen von Rivollis Bild möglich: die Flügel als Rettung Gottes vor dem Virus sowie eine Ankündigung der Apokalypse.

\section{Zwischen Leid und Hoffnung}

Ein weiterer, auf den ersten Blick vielleicht weniger expliziter Verweis auf die christliche Tradition findet sich in der Komposition des Bildes, die stark an andere Darstellungstypen von Maria erinnert. Dabei vereint das Bild zwei verschiedene Motive in sich: zum einen Maria mit dem Jesuskind. Dabei handelt es sich um eine Form der Mariendarstellung, die seit dem Hochmittelalter verbreitet ist und die Maria als Figur der Barmherzigkeit und der Zuflucht, die sich in mütterlicher Liebe zu den Menschen herabneigt, zeigt. Dadurch werden Fürsorge und Geborgenheit symbolisiert: Italien befindet sich in den Armen einer fürsorglichen Mutter. Diese Deutung wird von den Engelsflügeln unterstützt. Italien wird von einer als Maria mit Engelsflügeln stilisierten Ärztin versorgt, wodurch Hoffnung oder gar eine eschatologische Verheißung ausdrückt wird.

Zum anderen erinnert die Komposition an das Motiv der Pietà, der trauernden Maria mit dem toten Jesus im Arm (Abb. 11). Diese mater dolorosa, auf Deutsch auch als «Schmerzensmutter» bezeichnet, stellt einen Bezug zur Passionsgeschichte her und lädt die Betrachtenden ein, das Leiden Marias beim Anblick ihres verstorbenen Kindes mitzuerleben. Vor diesem Hintergrund betrachtet, hebt das Graffiti das Leiden der italienischen Nation in der Corona-Pandemie hervor. Italiens Silhouette ist blutrot, es liegt im Sterben. Die Nation schwebt zwischen Leid und Hoffnung, sie liegt im Sterben, aber auch in den fürsorglichen Armen einer Mutter - der Mutter Gottes. Auffällig ist, dass Italien den Platz einnimmt, an dem sich in Mariendarstellungen üblicherweise Jesus befindet. Dabei ist Italien zugleich der sterbende Jesus und das neugeborene Jesuskind. Die italienische Nation 


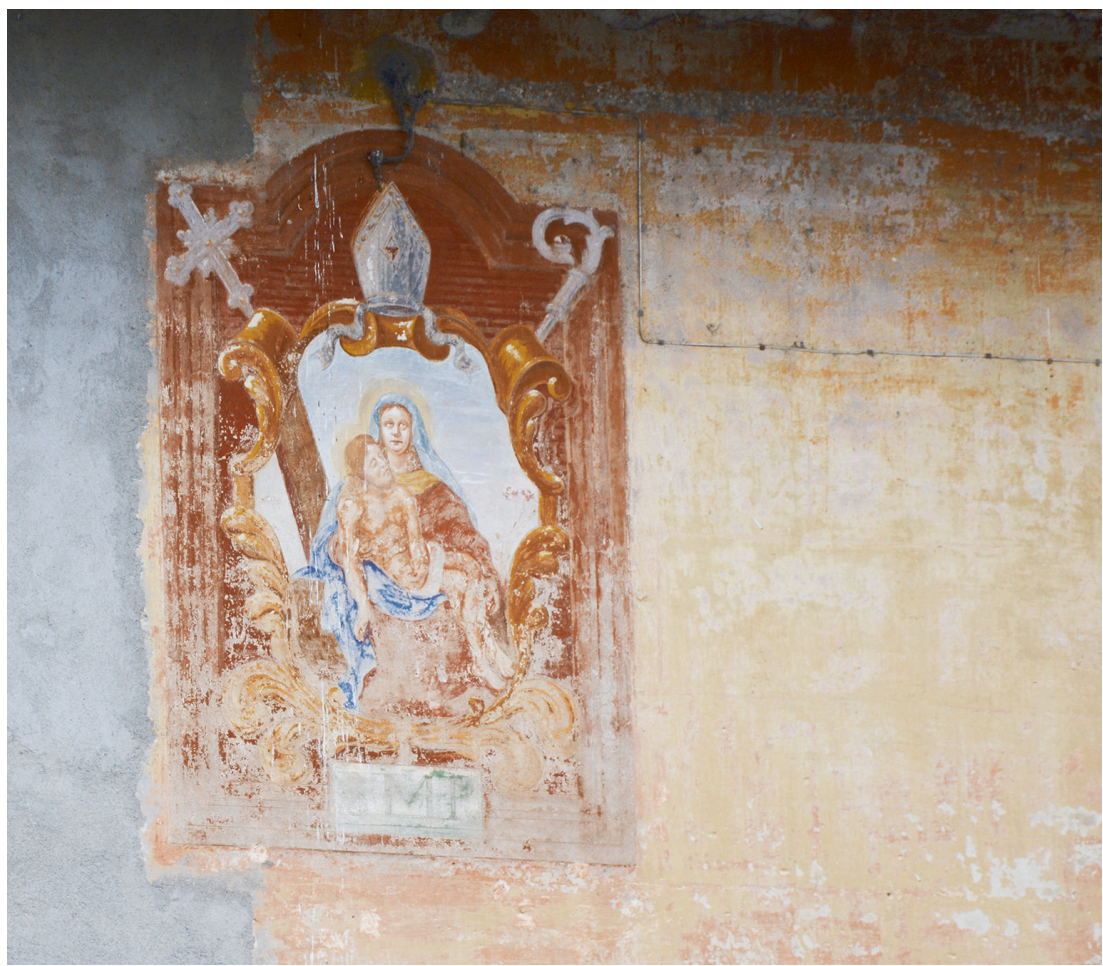

Abb. 11: Das Pietà-Motiv auf der Außenfassade eines Hauses in der Nähe der Stadt Mailand. Diese mittelalterliche Darstellung der trauernden Mutter Jesu ist bis heute im europäischen öffentlichen Raum weit verbreitet und wird in zahlreichen Stilen und Adaptionen dargestellt.

schwebt in dem Bild zwischen Leid, Todesdrohung und Hoffnung oder gar einem Heilsversprechen: Jesus ist auferstanden, diese Verheißung liegt auch für Italien im Bild.

Die Nation wird zugleich als Leidensgemeinschaft und als geborgen inszeniert. Die Figur der beflügelten Ärztin schwebt zwischen den Ikonographien der Mutter der Nation und der Mutter Jesu mit simultanen Verweisen auf Leiden und Hoffnung. Das Wandbild hebt dabei die nationale Gemeinschaft hervor, indem ausschließlich Italien dargestellt wird und nicht etwa Europa, die Welt oder nur die Lombardei oder Bergamo. Damit ist das Graffiti an Grenzziehungsprozessen beteiligt, indem es Italien als eine abgeschlossene Schicksalsgemeinschaft inszeniert. 
Das Bild enthält einen gleich vierfachen Verweis auf die italienische $\mathrm{Na}$ tion: Neben Stiefel und Flagge ist Blau Italiens Farbe im Sport. Zudem werden Nationen häufig als Frauen abgebildet. Insofern kann die Frau auf dem Bild auch als personifizierte Darstellung der italienischen Nation gelesen werden.

Angesichts der Deutungshorizonte, die sich anhand der möglichen Verweise auf Maria-Jesus-Kompositionen sowie die Personifikation der Nation eröffnen, wird der vielschichtige und zwiespältige Charakter des Bildes umso deutlicher.

\section{Zwischen Dank und Protest}

Als Bildgattungen des öffentlichen Raums leben Graffitis von ihrer Wechselbeziehung zum spezifischen Ort, an dem sie angebracht wurden. Dieser partikuläre Raum prägt die Bildwahrnehmung, ebenso wie das Kunstwerk die Wahrnehmung des Raumes beeinflusst. In diesem gegenseitigen Bezug entfaltet sich um das Bild ein Kommunikationsprozess, in dem vielfältige politische und religiöse Bedeutungen, Weltbilder und Konzepte verhandelt werden. Als öffentlich sichtbare Kunstwerke, die nicht zuletzt durch ihre Größe städtische Räume verändern, sind Graffitis privilegierte Medien, um gesellschaftspolitische Fragen aufzuwerfen.

Im Kontext der Corona-Krise stellen sie eine Form der Verarbeitung und Begleitung aktueller Geschehnisse dar. Aufgrund ihrer Entstehungsgeschichte ist diese spezifische Ausdrucksform eng mit Formen gesellschaftlichen Protests verbunden und weist einen kritischen Zug gegenüber bestehenden sozial-politischen Verhältnissen auf. In jedem Fall setzt ein solches Kunstwerk politische Kommunikationsprozesse in Gang und wirkt sich somit auf die gesamte Gesellschaft aus. Darüber hinaus «verlassen» Graffitis im Zuge ihrer medialen Reproduzierbarkeit die Straßen, indem sie fotografiert und über soziale Medien verbreitet werden. Damit wird der physische Stadtraum mit dem digitalen, medialen Raum verschränkt.

Damit wird ein weiterer Bedeutungshorizont von La dottoressa che culla l'Italia enthüllt: Obwohl das Wandbild ganz explizit Dank gegenüber dem Krankenhauspersonal ausdrückt, kann es auch als Kritik am italienischen Gesundheitssystem und damit am Umgang der Regierung mit der Pandemie und den Ressourcen gesehen werden. Indem es im öffentlichen Raum angebracht wurde und damit im Bereich des Staates, der gerade in der Krise seine Macht über diesen Raum in Form der Ausgangsbeschränkungen demonstriert hat, fordert das Kunstwerk die Deutungsmacht über den öffentlichen Raum für das Volk zurück. Dieser subversive Zug suggeriert so- 
mit eine Anklage an den Staat für das Leiden und Sterben des italienischen Volkes und beschwört die Souveränität, Solidarität und Zusammengehörigkeit der Bürger:innen.

\section{Zwischen Tod und Erlösung}

Durch die mediale Verbreitung und die Verschmelzung des Wandbildes mit seinen unzähligen Reproduktionen wird das Graffiti in Bergamo zum Sinnbild des gesamten öffentlichen, nationalen Raums, das eine allgemeine italienische Identität prägt. Dies ist umso bemerkenswerter, als der öffentliche Raum in der Corona-Krise nur beschränkt zugänglich ist. Der mediale Raum ersetzt in der Pandemie die begehbaren öffentlichen Orte der Gesellschaft. Somit macht das Kunstwerk das Krankenhaus und Bergamo zum symbolischen Ort der Corona-Krise, an dem sich das Schicksal der italienischen Nation entscheidet. In dem Wandbild und seiner medialen Verbreitung verschränken sich begehbarer und medialer öffentlicher Raum. Der begehbare wird in den medialen Raum eingebettet und der mediale Raum dient zur Beanspruchung und Bedeutungszuschreibung des begehbaren Raums. In der Folge verschwimmt im Foto-Graffiti die Wahrnehmung der beiden Dimensionen des öffentlichen Raums. Der nationale Identitätsraum Italiens wird somit auf den medialen Raum ausgeweitet.

In diesem Kontext spielt die Mehrdeutigkeit des Bildes eine wesentliche Rolle: Die Darstellung verbindet Schutz und Apokalypse, Leid und Trost, drohenden Tod und Hoffnung auf Erlösung. Je nach Betrachtungsweise rückt die eine oder die andere Bedeutung in den Vordergrund. Dabei können Elemente des Kontextes die jeweiligen Deutungsweisen befördern. Der Schriftzug, der Dank gegenüber dem Krankenhauspersonal zum Ausdruck bringt sowie die fürsorgliche Handhaltung der Ärztin können den Aspekt der Hoffnung unterstützen. Die Kleidung der Intensivstation und die blutrote Farbe des italienischen Stiefels wiederum betonen Tod und Leid. Im Wandbild sind vielfältige Bedeutungen angelegt. Ihre letztendliche Deutung liegt bei den Betrachtenden.

Das Graffiti greift auf ein christliches, gesellschaftlich geteiltes Bildrepertoire zurück. Die Verweise auf religiöse Motive unterstützen die Deutungen des Leidens und der Hoffnung Italiens im Horizont der christlichen Heilsgeschichte. Das Leid und die Hoffnung Italiens sowie die gesamte Nation werden dadurch in einen größeren und religiösen Bedeutungszusammenhang gestellt. Dadurch wird der Corona-Krise eine Art Sinnhaftigkeit zugeschrieben, indem die Anspielung auf die christliche Heilsgeschichte dem Leid einen Sinn verleiht und Erlösung verspricht. Indem das Wand- 
bild religiös konnotierte Motive und Narrative hervorruft, die im kollektiven Gedächtnis verankert sind, entfaltet es eine starke emotionale Wirkung und erlangt Glaubwürdigkeit: Die Ärztin, die Italien wiegt, wird damit zur nationalen Ikone der Krise stilisiert.

Literatur

Glaser, Katja, 2017, Street Art und neue Medien. Akteure - Praktiken - Ästhetiken, Bielefeld: transcript.

Hipfl, Brigitte, 2004, Medien als Konstrukteure (trans-)nationaler Identitätsräume, in: Hipfl, Brigitte/Klaus, Elisabeth/Scheer, Uta (Hg.), Identitätsräume. Nation, Körper und Geschlecht in den Medien. Eine Topografie, Bielefeld: transcript, 53-59.

Klee, Andreas, 2010, Graffiti als Medium des Politischen?!, in: ders. (Hg.), Politische Kommunikation im städtischen Raum am Beispiel Graffiti, Wiesbaden: VS Verlag für Sozialwissenschaften, 109-119.

Klitzke, Katrin/Schmidt, Andreas, 2009, Steet Art. Legenden zur Straße, Berlin: Archiv der Jugendkulturen Verl.

Pezzoli-Olgiati, Daria, 2016, Sichtbare Religion. Bilder, Blicke und Visualität als Grundthemen der Religionswissenschaft, SAGW Akademievortrag, Bern: Schweizer Akademie der Geistes- und Sozialwissenschaften.

Schmidt, Heinrich/Schmidt Margarethe, 2018 (1981), Die vergessene Bildersprache christlicher Kunst. Ein Führer zum Verständnis der Tier-, Engel- und Mariensymbolik, München: C.H. Beck, 2. Aufl. 